\title{
Guest Editorial: Introduction to Philosophical Issues in Neuroethics
}

TUIJA TAKALA

Neuroethics studies the ethical, social, and legal issues raised by actual or expected advances in neuroscience. The relevant fields in neuroscience include, but are not limited to, neuroimaging, cognitive neuroscience, neuropsychopharmacology, neurogenetics, and neuropsychiatry. For many, neuroethics is best understood as a subcategory of bioethics, and although not all agree, for the purposes of the present collection of articles, this definition is assumed. Although bioethics as a field of study started in the early 1970s as a normative enterprise, mainly practiced by philosophers and theologians, it has since become truly inter- and multidisciplinary, comprising also law, sociology, psychology, gender studies, disability studies, anthropology, history, and many other approaches. What we particularly wanted to do in this issue, however, was to find the most pertinent questions of neuroethics from the viewpoint of philosophy, and the call for papers was drafted accordingly.

In the call, contributors were asked to address philosophical issues in neuroethics or to analyze neuroethical problems philosophically. Very quickly it became obvious that most authors found the issue of moral responsibility and related problems as both the most challenging and the most important for philosophical neuroethics. The questions of moral responsibility in the light of biomedical findings are not new to bioethics-they have been discussed widely in relation to, for instance, genetic testing-but the possibilities of understanding how the brain works seem to take the challenge a step further. Problems of the philosophy of mind can no longer be avoided, and wherever one looks the issue of free will seems to surface. Four of the articles address these issues.

Another ethically and philosophically relevant aspect that neurosciences share with genetics is the often uncertain and, for the most part, only statistically relevant nature of the data that are acquired by the various imaging and testing techniques (at least in the light of current scientific understanding). This is especially significant when discussing the normative questions of what to do with such findings. In relation to moral responsibility, the questions encountered are frequently linked with unwelcome behavior, or the risk of it, and whether individuals who appear to show dispositions of such behavior should be treated differently. In addition to the difficult normative dilemmas, the study of these

The editorial work for this special issue was supported by my project Neuroscience and Norms: Ethical and Legal Aspects of Norms in Neuroimaging (NeuroSCAN) / Ethical Concepts and Norms funded by the Academy of Finland (SA 1124638). 
issues requires philosophical analysis of notions like dispositions and likelihoods and logical scrutiny of the use of statistical data.

In the current regulation-happy climate, philosophical studies often cannot avoid making occasional references to the law, either as it is or as it ought to be. Rather than leading us to think that ethics and law are becoming one, the hope is that the articles of this special section show the philosophical complexity of the matters behind laws that regulate the use and applications of bioscientific advances.

The first article is Matti Häyry's overview of philosophical approaches to neuroethics. Häyry starts by identifying the practices of neuroscience that are causing, and will increasingly continue to cause, ethical problems. According to him these are brain imaging, chemical attempts to shift exceptional brain function towards normality (curing), chemical attempts to enhance cognitive functioning beyond what is considered normal (enhancement), and other methods of manipulating the brain. Häyry then draws our attention to the core philosophical problems of mind and matter, thinking and the brain, and freedom and determination that we cannot avoid when doing neuroethics. In the latter half of his paper, Häyry introduces us to the main philosophical theories (and antitheories) that can be used in attempts to address the ethical and philosophical problems raised by advances in neurosciences. Although he is skeptical as to whether any theoretical approach in and by itself could provide the answers, he maintains that philosophical analysis is needed to gain a deeper understanding of the issues and perhaps even to help us toward the (right) solutions.

The first two papers of the four dealing with moral responsibility draw partly from the philosophy of science to offer a critical and somewhat cautious view to making use of neuroscientific data when considering criminal and moral responsibility. Matthew R. Broome, Lisa Bortolotti, and Matteo Mameli think that it is too early to know how advances in the neurosciences will ultimately impact our notion of moral responsibility and that consequently we should not, for the time being, worry about the notion's survival. What they see as important, however, is that we use the advances in these sciences to study the current notion of moral responsibility and its distinctions and criteria. Through a case study they look at the implications that discoveries in neuropsychology and neuropsychiatry can have to the way that moral responsibility is attributed to agents with and without diagnoses of mental illness. Their analysis shows that simplistic labels like "mentally ill" are insufficient in attributing moral or legal responsibility, and that a more nuanced way of understanding behavior (both normal and pathological) is needed.

In his article, Gardar Árnason considers the methodological questions of neuroimaging, especially functional magnetic resonance imaging (fMRI), and its implications on our judgments concerning people's liability and culpability in courts of law. He argues that, because of unresolved issues with the reliability and validity of neuroimaging studies, these studies should be used with considerable caution in the criminal justice system. Scientific findings come with uncertainties, probabilities, and caveats that tend to be forgotten when neuroscientific advances are popularized and applied to social and legal issues. There are, however, legitimate pressures that will probably increase the use of neuroimaging evidence in trials in the near future. Árnason welcomes applications of fMRI and other forms of neuroimaging as long as they are not seen to prove more than what is scientifically warranted, but warns that temptations to go beyond justifiable interpretations are likely to be strong. 


\section{Guest Editorial}

The paper by Tom Buller makes use of philosophy of mind to show us how our deeply rooted and, for many purposes necessary, assumption of free will can survive advances in neuroscience. In his contribution, Buller argues that, contrary to what some authors (he is mainly looking at arguments put forward by Jonathan Greene and Joshua Cohen) have claimed, empirical evidence cannot be used to disprove the existence of free will, because empirical evidence only tells us what is, not what has to be. Also, determinism at the neurobiological level does not necessarily mean determinism, say, on the level of cognition. If Buller is right, this means that, against widely held beliefs, neuroscience can never provide data that would, as such, invalidate free will.

Sabine Müller and Henrik Walter start by showing how the notion of autonomy currently prevailing in bioethics does not adequately take into account lessons from philosophy of mind and from neuroscience. In their paper they suggest that freedom from internal coercive influences requires more attention when a person's autonomy, or the lack of it, is evaluated. Using a concept of "natural autonomy" that was recently introduced to the free will debate and puts special emphasis on internal constraints, they show how neuroscience can help to determine the degree of a person's autonomy. In the justification of their thesis, they use as examples the deep brain stimulation of the subthalamic nucleus and the body integrity identity disorder. Although they do not discuss this at any great length, another aspect worth noting here is that the notion of "natural autonomy" can also be seen as a solution to the problem of free will, as it is not rooted in "indeterministic processes."

The final article approaches neuroethics from the viewpoint of argumentation analysis. In his contribution, Veikko Launis studies the use of slippery slope arguments in discussions on the ethics of cosmetic neurology. For a variety of reasons, he finds that both the empirical and conceptual versions of the argument are unable to provide us with conclusive moral reasons for banning moderate cosmetic neurology. A less frequently used variation of the argument, sometimes called the "arbitrary result slippery slope argument," is the one that Launis finds the most interesting and the most important. Although this version does not seek to ban anything conclusively, he sees its strength in that it reminds us to be as specific and as consistent as possible about our basic moral, medical, and scientific concepts.

The six articles of this special section address some of the key issues of philosophical neuroethics, but there are many topics, themes, and perspectives that merit further study. I hope that the readers will not only find these articles useful, but that they will also find in the views and arguments presented an incentive to become involved in neuroethical studies, especially in the philosophical aspects of the field.

On a broader note, philosophical bioethics has, of late, struggled to find its proper role alongside practical, regulatory, and multidisciplinary bioethics. Neuroethics has given conceptual studies a new lease of life, as issues in neuroethics simply cannot be approached without considerable input from philosophy of mind and philosophy of science, just to mention two of the many fields of philosophy that are relevant here. With its newfound recognition in the context of neuroethics, philosophy could, it is to be hoped, also regain some of its legimate ground in bioethics more generally. 